\author{
MITSUBISHI ELECTRIC RESEARCH LABORATORIES \\ http://www.merl.com
}

\title{
Sparse MIMO Architectures For Through-The-Wall Imaging
}

\author{
Li, L; Boufounos, P.T.; Liu, D.; Mansour, H.; Sahinoglu, S.
}

TR2014-051 June 2014

\begin{abstract}
Compressive sensing and sparse array processing has provided new approaches to improve radar imaging systems. This paper, explores the potential of sparse Multiple-Input-Multiple-Output (MIMO) radar arrays to significantly reduce the cost of through-the-wall imaging (TWI). We analyze three well-known sparse array structures-nested arrays, co-prime arrays and random arrays-and examine their performance in the presence of common types of layered walls. The reconstruction is performed by formulating and solving a wall parameter estimation problem in conjunction with a sparse reconstruction problem that takes the wall parameters into account. Our simulation results demonstrate the effectiveness of our approach and validate the performance of the system for the three different MIMO sparse array structures.
\end{abstract}

IEEE Sensor Array and Multichannel Signal Processing Workshop (SAM), 2014

This work may not be copied or reproduced in whole or in part for any commercial purpose. Permission to copy in whole or in part without payment of fee is granted for nonprofit educational and research purposes provided that all such whole or partial copies include the following: a notice that such copying is by permission of Mitsubishi Electric Research Laboratories, Inc.; an acknowledgment of the authors and individual contributions to the work; and all applicable portions of the copyright notice. Copying, reproduction, or republishing for any other purpose shall require a license with payment of fee to Mitsubishi Electric Research Laboratories, Inc. All rights reserved. 



\title{
Sparse MIMO Architectures For Through-The-Wall Imaging
}

\author{
$\mathrm{Li} \mathrm{Li}^{*}$, Petros T. Boufounos ${ }^{\dagger}$, Dehong $\mathrm{Liu}^{\dagger}$, Hassan Mansour ${ }^{\dagger}$, and Zafer Sahinoglu ${ }^{\dagger}$ \\ * Department of Electrical and Computer Engineering, Duke University, Durham, NC \\ ${ }^{\dagger}$ Mitsubishi Electric Research Laboratories (MERL), Cambridge, MA
}

\begin{abstract}
Compressive sensing and sparse array processing has provided new approaches to improve radar imaging systems. This paper, explores the potential of sparse Multiple-Input-Multiple-Output (MIMO) radar arrays to significantly reduce the cost of through-the-wall imaging (TWI). We analyze three well-known sparse array structures-nested arrays, co-prime arrays and random arrays-and examine their performance in the presence of common types of layered walls. The reconstruction is performed by formulating and solving a wall parameter estimation problem in conjunction with a sparse reconstruction problem that takes the wall parameters into account. Our simulation results demonstrate the effectiveness of our approach and validate the performance of the system for the three different MIMO sparse array structures.
\end{abstract}

Index Terms-Through-the-wall, MIMO sparse arrays, Sparse image reconstruction, compressive sensing.

\section{INTRODUCTION}

The recent success of compressive sensing and other sub-Nyquist sampling and acquisition methods has reinvigorated interest in sparse, undersampled radar arrays [1]-[3]. These arrays enable radar signal acquisition and imaging using significantly fewer array elements compared to conventional array structures, thus significantly reducing the array implementation cost.

Sparse arrays have average inter-element spacing much larger than half the wavelength of the transmitted signal, which is the Nyquist interval for array processing. This is achieved using non-uniform element spacing, which eliminates fundamentally unresolvable ambiguities, known as grating lobes. While classical algorithms have been used in the past to recover the acquired image, they suffer from the increased sidelobes exhibited by those arrays. However, recently developed sparse recovery algorithms are robust to sidelobes, thus enabling imaging using significantly fewer array elements.

In this work, we explore the application of sparse Multi-InputMulti-Output (MIMO) arrays and Compressive Sensing (CS) for through-the-wall imaging (TWI). MIMO architectures exhibit reduced array gain due to waveform diversity, yet they provide finer spatial resolution, more degrees of freedom, improved performance in parameter identifiability, and multipath rejection [4], [5]. Most work on CS-based TWI modifies the acquisition hardware [6], [7]. Instead, similar to [8], we use sparse arrays to reduce the acquisition cost.

Specifically, this paper explores and compares three well-known sparse array structures: co-prime [9], nested [10] and uniformly random arrays [3], in the presence of layered lossless walls. Under the assumption that the imaged scene is sparse, we analyze imaging performance of different sparse array architectures and wall profiles in terms of mainlobe and sidelobe structure, i.e., by examining the characteristics of the point spread function (PSF). The PSF, known as beampattern in the array literature, is intimately related to the mutual coherence in the context of the sparse recovery literature [11]. The PSF characteristics provide good intuition on the performance of the array both under conventional and sparse reconstruction algorithms.

Since the increased sidelobe levels of the proposed architectures decrease the performance of conventional imaging algorithms, we

Li Li was with MERL when this work was conducted.

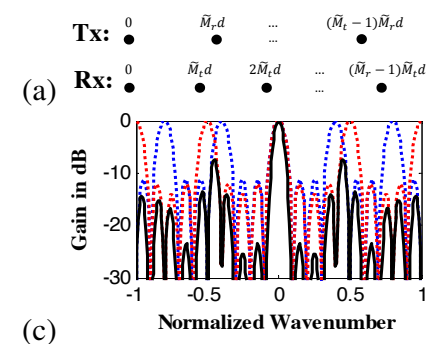

(b)

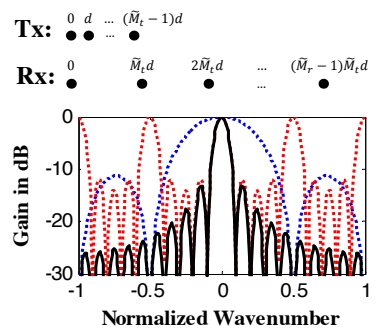

Fig. 1. Sparse array structures (top) and sample corresponding beampatterns (bottom): co-prime (left) and nested arrays (right). Blue shows the transmitted, red shows the received, and black shows the total product beampatterns.

use sparse reconstruction approaches to exploit the sparsity of the scene of interest. In particular, we apply Iterative Hard Thresholding (IHT) [12]—a well-established greedy-based sparse signal recovery algorithm - to estimate the reflectivity map behind the wall. Furthermore, we develop an algorithm to estimate the parameters of the wall profile from the received data. These parameters are used to develop imaging operators necessary in the reconstruction algorithm.

\section{TWI SIGNAL MODEL}

In this work, we assume a 2D imaging scenario, where the MIMO radar array is located at the origin with a $d_{0}$ standoff distance from the wall. The positions of the $M_{t}$ transmitter (Tx) and $M_{r}$ receiver (Rx) elements are denoted using $\mathbf{t}_{i}, i=1, \ldots, M_{t}$ and $\mathbf{r}_{i}, i=$ $1, \ldots, M_{r}$, respectively. Using the point target approximation, the received scattered field in the frequency domain, excluding effects of direct wall reflections and additive observation noise, equals [13]:

$$
y(\mathbf{t}, \mathbf{r}, \omega) \cong \int_{S} s(\mathbf{p}) w(\omega) g(\mathbf{r}, \mathbf{p}, \omega) g(\mathbf{t}, \mathbf{p}, \omega) d \mathbf{p} .
$$

In (1), $w(\omega)$ represents the frequency signature of the transmitted radar waveform, $s(\mathbf{p})$ denotes the reflectivity of the object of interest located at $\mathbf{p}=(x, y)$, and $S$ denotes the surveyed region. The function $g\left(\mathbf{p}_{1}, \mathbf{p}_{2}, \omega\right)$ denotes the Green's function for a layered medium from point $\mathbf{p}_{1}$ to $\mathbf{p}_{2}$ [14], which is a function of the thickness, $d$, and relative permittivity, $\epsilon$, of the wall, and incorporates the multiple reflections due to the wall [15].

To discretize the system, we divide the observed region $S$ using a grid of $P$ points, and represent the complex reflectivity of the map using $\mathbf{s} \in \mathbb{C}^{(P \times 1)}$. Assuming $N$ frequency samples are obtained at each $\mathrm{Rx}$ element, the discretized version of (1) is given by:

$$
\begin{aligned}
& \mathbf{y}=\mathbf{\Phi} \mathbf{s} \\
& \text { where } \\
& \left.\qquad \begin{array}{c}
\mathbf{y}=\left[y\left(\mathbf{t}_{1}, \mathbf{r}_{1}, \omega_{1}\right), y\left(\mathbf{t}_{1}, \mathbf{r}_{1}, \omega_{2}\right), \ldots, y\left(\mathbf{t}_{M_{t}}, \mathbf{r}_{M_{r}}, \omega_{N}\right)\right]^{T}, \\
\mathbf{\Phi =}=\left[\phi_{1}, \phi_{2}, \ldots, \phi_{P}\right] \in \mathbb{C}^{\left(M_{r} M_{t} \times P\right)} \\
w\left(\omega_{1}\right) g\left(\mathbf{r}_{1}, \mathbf{p}_{i}, \omega_{1}\right) g\left(\mathbf{t}_{1}, \mathbf{p}_{i}, \omega_{1}\right) \\
w\left(\omega_{2}\right) g\left(\mathbf{r}_{1}, \mathbf{p}_{i}, \omega_{2}\right) g\left(\mathbf{t}_{1}, \mathbf{p}_{i}, \omega_{2}\right) \\
\vdots \\
w\left(\omega_{N}\right) g\left(\mathbf{r}_{M_{r}}, \mathbf{p}_{i}, \omega_{N}\right) g\left(\mathbf{t}_{M_{t}}, \mathbf{p}_{i}, \omega_{N}\right)
\end{array}\right],
\end{aligned}
$$

where $\mathbf{y}, \phi_{i} \in \mathbb{C}^{\left(M_{r} M_{t} N \times 1\right)}$ and $\boldsymbol{\Phi}$ is the array manifold matrix. 


\section{SPARSE ARray DESIGN}

\section{A. Sparse Array Architectures}

Our sparse array design starts with a notional grid of $M_{r}$ and $M_{t}$ uniformly spaced possible Tx and Rx elements, respectively. We subsample the grid according to each architecture-co-prime, nested or random. Only a few grid points include actual Tx or Rx elements.

Co-prime arrays [14] are defined by a pair of co-prime numbers $\widetilde{M}_{t}$ and $\widetilde{M}_{r}$, for Tx and Rx arrays, respectively. The Tx array consists of $\widetilde{M}_{t}$ sensors with an inter-element spacing of $\widetilde{M}_{r}$ grid units, while the Rx array consists of $\widetilde{M}_{r}$ elements with an inter-element spacing of $\widetilde{M}_{t}$ grid units, as shown in Fig. 1 (a). Nested arrays [16] also comprise of two uniform linear arrays (ULA); the Tx array consists $\widetilde{M}_{t}$ sensors with spacing of one grid unit, while the Rx consists $\widetilde{M}_{r}$ elements with spacing of $\widetilde{M}_{t}$ units, as shown in Fig. 1 (b). Random arrays with the same aperture are designed by randomly selecting $\widetilde{M}_{t}$ Tx and $\widetilde{M}_{r}$ Rx elements from each grid using a uniform distribution.

The optimal MIMO sparse nested array can be obtained by maximizing the degrees of freedom $\widetilde{M}_{r} \widetilde{M}_{t}$, given the total number of MIMO elements $\widetilde{M}_{r}+\widetilde{M}_{t}$. For a co-prime array, an additive prime constraint should also be included. Examples of MIMO array beam pattern for co-prime array and nested array with $\widetilde{M}_{r}=4$ and $\widetilde{M}_{t}=$ 5 are shown in Fig. 1(c) and (d), respectively.

The $\widetilde{M}_{r} \times \widetilde{M}_{t}$ sparse MIMO array can be considered a subsampling of a $M_{r} \times M_{t}$ full MIMO array. This can be represented using a subsampling matrix $\mathbf{D} \in\{0,1\}^{\widetilde{M}_{r} \widetilde{M}_{t} N \times M_{r} M_{t} N}$. Using $\boldsymbol{\Phi}$ and $\widetilde{\boldsymbol{\Phi}}$ to denote the manifold matrices of the full and sparse arrays, respectively, the acquisition function (2) for the sparse array becomes

$$
\widetilde{\mathbf{y}}=\mathbf{D} \boldsymbol{\Phi} \mathbf{s}=\widetilde{\boldsymbol{\Phi}} \mathbf{s},
$$

where $\widetilde{\mathbf{y}}$ denoting the subsampled received data.

\section{B. Array Design Properties}

When considering the properties of an array design, classical array literature focuses on the PSF, or beampattern, of the array. Furthermore, it is well understood (e.g., see [1]) that the PSF, appropriately normalized, is equivalent to the mutual coherence between columns of the manifold matrix, a key property of interest in CS acquisition systems. The coherence between two columns is defined as the normalized inner product between them, whereas the coherence of the matrix is defined as the maximum absolute value of this inner product among all pairs of elements in the matrix [11].

$$
\mu(\widetilde{\boldsymbol{\Phi}})=\max _{i \neq j} \frac{\left|\widetilde{\phi}_{j}^{H} \widetilde{\phi}_{i}\right|}{\left\|\widetilde{\phi}_{i}\right\|_{2}\left\|\widetilde{\phi}_{j}\right\|_{2}} .
$$

A low matrix coherence $\mu(\widetilde{\boldsymbol{\Phi}})$ is sufficient but not necessary to provide worst-case sparse reconstruction guarantees. On the other hand, the coherence structure described by the PSF, $\widetilde{\boldsymbol{\Phi}}^{H} \widetilde{\phi}_{i}$, provides significantly more information about the performance of the array, especially under classical algorithms, such as the resolution, the noise and interference robustness, and the points in the observed area that can potentially cause reconstruction ambiguities.

The figures of merit we consider are the mainlobe area (MLA) and the maximum sidelobe level (MSL). The MLA is defined as the area around a point in the scene for which the PSF is above a certain level, typically $-3 \mathrm{~dB}$. The MSL is defined as the highest level the PSF reaches in its sidelobes, i.e., outside of the main lobe. The MLA is a measure of the array resolution, since it represents the ambiguity around a scene point. The MSL is a measure of the recoverability of a particular scene point, since it measures the maximum mutual coherence of that point with the other points in the scene.

\section{SCENE RECONSTRUCTION}

\section{A. Wall profile estimation}

To compute the Green's function in (1) it is necessary to know the permitivity $\epsilon_{l}$ and the thickness $d_{l}, l=1, \ldots, L$, for all $L$ layers of the layered wall. Since the wall is not known in advance, we estimate these from the acquired data, assuming $L$ is known.

Excluding self-coupling between Tx and Rx elements, the received return from all $\mathrm{Rx}$ elements consists of multipath components from each wall layer. We assume the bistatic $\mathrm{Tx}$ and $\mathrm{Rx}$ element are separated by $\Delta=\|\mathbf{r}-\mathbf{t}\|$ but share the same standoff distance $d_{0}$ from the wall. Using Snell's law, the reflection from the $l^{\text {th }}$ layer arrives with delay $\tau_{l}(\Delta)$, i.e.. time of arrival (TOA) equal to

with

$$
\tau_{l}(\Delta)=\frac{2}{c} \sum_{i=1}^{l} \epsilon_{i} r_{i}
$$

$$
\begin{gathered}
\sum_{i=i}^{l} \sqrt{r_{i}^{2}-d_{i}^{2}}=\frac{\Delta}{2} \\
\epsilon_{i-1} \frac{r_{i-1}^{2}-d_{i-1}^{2}}{r_{i-1}^{2}}=\epsilon_{i} \frac{r_{i}^{2}-d_{i}^{2}}{r_{i}^{2}}, i=2, \ldots, l
\end{gathered}
$$

where the $r_{i}$ is the one-way traveling distance within each layer.

Thus, the unknown wall parameters, collectively denoted using $\theta=\left\{\epsilon_{1}, \ldots, \epsilon_{L}, d_{1}, \ldots, d_{L}\right\}$, can be obtained by minimizing the mean squared error between the measured TOA of each reflection, $\tau_{l}(\Delta), l=1, \ldots L$ and the predicted TOA $\widehat{\tau}_{l}(\Delta, \theta)$ given the wall parameters from each layer:

$$
\theta=\arg \min _{\theta} \sum_{l=1}^{L} \sum_{j=1}^{\widetilde{M}_{t} \widetilde{M}_{r}} \alpha_{j}\left|\tau_{l}\left(\Delta_{j}\right)-\widehat{\tau}_{l}\left(\Delta_{j}, \theta\right)\right|^{2}
$$

where $\alpha_{j}$ is the weight assigned to each Tx-Rx separation and $\widetilde{M}_{t} \widetilde{M}_{r}$ is the total number of Tx-Rx separations from the array. For limited bandwidth or low SNR applications, more accurate TOA estimates can be obtained using super-resolution or adaptive techniques [16].

\section{B. Sparse Image Recovery}

To recover the scene reflectivity $\widehat{\mathbf{s}}$ from the measurements $\widetilde{\mathbf{y}}$ in (6) we assume the scene is sparse and use standard CS techniques. Specifically, we formulate the sparsity constrained minimization

$$
\widehat{\mathbf{s}}=\arg \min _{s}\|\widetilde{\mathbf{y}}-\widetilde{\mathbf{\Phi}} \mathbf{s}\|_{2}^{2} \text { s.t. }\|\mathbf{s}\|_{0} \leq K,
$$

where $K$ is the maximum sparsity of s, i.e., the maximum number of reflectors in the discretized scene. While in general the problem is NP-hard, it can often be solved by relaxing the $\ell_{0}$ norm to its $\ell_{1}$ convex hull or using one of the many available greedy algorithms, e.g., [12], [17], [18]. In this work we use Iterative Hard-thresholding (IHT) [12], an iterative algorithm in which the sparse estimate $\widehat{\mathbf{s}}_{\Omega}^{(t+1)}$ at iteration $t$ is estimated using

$$
\widehat{\mathbf{s}}_{\Omega}^{(t+1)}=\mathcal{T}_{K}\left(\widehat{\mathbf{s}}_{\Omega}^{(t)}+\eta \widetilde{\boldsymbol{\Phi}}^{H}\left(\widetilde{\mathbf{y}}-\widetilde{\boldsymbol{\Phi}} \widehat{\mathbf{s}}^{(t)}\right)\right),
$$

where $\eta$ is a step size, and $\mathcal{T}_{K}(\cdot)$ is a hard thresholding operator that preserves the $K$ largest in magnitude components of its argument and sets the remaining components to 0 . We prefer the IHT because, in our experiments, it provides a great balance of computational cost and recovery performance compared to alternatives. It also allows for greater adaptability to signal models using model-based CS [19]. The IHT can be further accelerated using techniques explored in detail in [20], [21], e.g., by adapting the step-size selection in each iteration.

\section{Simulation Results}

\section{A. Evaluation of Array Designs}

Our first set of experiments examines the PSF properties of the array designs under consideration. Specifically, we consider a $5 \mathrm{~m} \times$ 


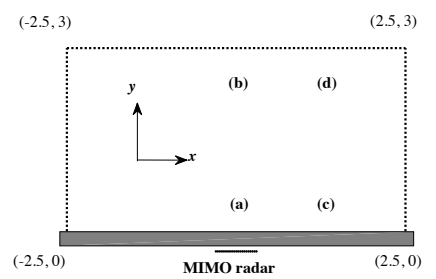

Fig. 2. Experimental setup to evaluate array designs
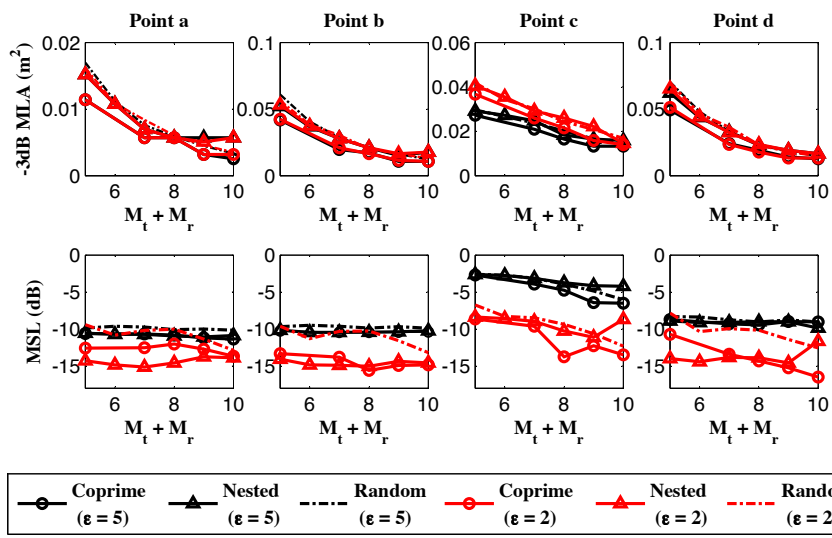

\begin{tabular}{cc} 
Rested & Random \\
$(\varepsilon=5)$ & $-\cdots 5)$ \\
\hline
\end{tabular}

Coprime
$(\varepsilon=2)$

$\begin{gathered}\text { Nested } \\ (\varepsilon=2)\end{gathered}-\cdot \begin{gathered}\text { Random } \\ (\varepsilon=2)\end{gathered}$

Fig. 4. Evaluation of array properties for 4 different scene points

$3 \mathrm{~m}]$ scene, as shown in Fig. 2. The phase center of the MIMO radar is located at the origin. In front of the array there is a single layer slab wall with thickness $d=0.1 \mathrm{~m}$. We examine four representative points of interest in the scene, at locations [0, 0.7], [0, 2.5], [1.2, 0.7] and $[1.2,2.5]$, denoted as points $\mathrm{a}, \mathrm{b}, \mathrm{c}$ and $\mathrm{d}$ in Fig. 2.

The PSFs of the four points of interest are shown in Fig. 3 for a wall with relative permittivity (a) $\epsilon=2$ and (b) $\epsilon=5$, for the co-prime (top), nested (middle) and a single realization of the random (bottom) array. All arrays have $\widetilde{M}_{t}=4$ and $\widetilde{M}_{r}=5$. Red and blue crosses locate the peak positions of the mainlobe and maximum sidelobe. As evident in the figure, each configuration exhibits very different characteristics, severely affected by the wall profile. In general, nested arrays exhibit better robustness to wall properties.

Fig. 4 illustrate the $-3 \mathrm{~dB}$ MLA in $\mathrm{m}^{2}$ and MSL in $\mathrm{dB}$ for the different configurations different sparse arrays as a function of the number of MIMO elements $\widetilde{M}_{r}+\widehat{M}_{t}$ with $\epsilon=2$ and 5. The MLAs and MSLs of the random arrays are computed by averaging over 50 realizations of array geometries for each value of $\widetilde{M}_{r}+\widetilde{M}_{t}$.

From Fig. 4 we can draw the following conclusions: i) Co-prime arrays have better cross-range resolution (measured by MLA) than nested arrays since co-prime arrays provide larger virtual aperture length. In contrast, nested arrays exhibit lower MSL. ii) Random arrays in general produce higher MSLs compared to co-prime and nested arrays. iii) The effect of array geometry on MSL is significantly diminished for walls with higher relative permittivity; overall, MSL increases as relative permittivity increases. iv) For smaller wall permittivity, multiple reflections may cause ambiguities in the range profile resulting in enlarged MLAs. For larger wall permittivity, multiple reflections produce better range resolvability with lower MLA but larger MSL. v) Points of interest near the endfire (e.g., see scene point c) suffer more serious influence from wall multiples, since the Fresnel reflection coefficient of the air-wall interface increases.

\section{B. Image Recovery}

While PSF characteristics are important in the system design, they do not convey the whole picture. For that reason, we also performed
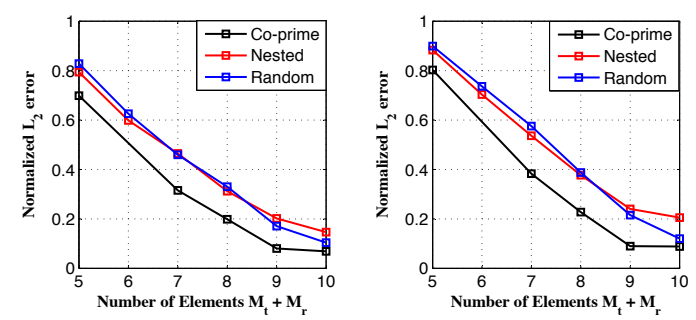

Fig. 5. Reconstruction error for the three array architectures

\begin{tabular}{|c|c|c|c|c|}
\hline & $0 \mathrm{~dB}$ & $5 \mathrm{~dB}$ & $10 \mathrm{~dB}$ & Truth \\
\hline$d_{1}$ & $5.56 \pm 0.29$ & $5.30 \pm 0.44$ & $5.25 \pm 0.22$ & 5.35 \\
\hline$\epsilon_{1}$ & $4.54 \pm 0.43$ & $5.01 \pm 1.02$ & $5.97 \pm 0.54$ & 5 \\
\hline$d_{2}$ & $5.75 \pm 0.33$ & $5.97 \pm 0.54$ & $6.24 \pm 0.46$ & 6.25 \\
\hline$\epsilon_{2}$ & $11.74 \pm 1.34$ & $11.06 \pm 1.73$ & $10.06 \pm 1.40$ & 10 \\
\hline$d_{3}$ & $3.67 \pm 0.48$ & $3.92 \pm 0.33$ & $3.93 \pm 0.14$ & 3.75 \\
\hline$\epsilon_{3}$ & $5.58 \pm 1.67$ & $4.75 \pm 0.96$ & $4.62 \pm 0.30$ & 5 \\
\hline \multicolumn{4}{|c}{ TABLE I }
\end{tabular}

TOA BASED WALL PROFILE ESTIMATION RESULTS FROM THE FDTD SIMULATION WITH 30 NOISE REALIZATIONS

two sets of image recovery experiments.

In the first set of experiments, we generate the exact MIMO sensing matrix $\widetilde{\boldsymbol{\Phi}}$, with known wall parameters. We consider a $3 \mathrm{~m} \times 3 \mathrm{~m}$ region of interest where the phase center of the MIMO radar located at $[0,0]$, with a zero standoff distance from a single layer slab wall with thickness $d=0.1 \mathrm{~m}$. The radar uses a pulse bandwidth from $3 \mathrm{GHz}$ to $5 \mathrm{GHz}$. We start with full MIMO Tx and Rx ULA with 21 elements each, having inter-element spacing $3 \mathrm{~cm}$. The scene is discretized to a $32 \times 32$ grid. We generated random reflectivity maps with $K=35$ point reflectors randomly placed on the grid, with standard normal reflectivity distribution. To evaluate performance we measure the $\ell_{2}$ reconstruction error $\|\widehat{\mathbf{s}}-\mathbf{s}\|_{2} /\|\mathbf{s}\|_{2}$.

Figure 5 plots the reconstruction error for the three array architectures as a function of the total sparse array elements $\widetilde{M}_{r}+\widetilde{M}_{t}$ with relative wall permittivity $\epsilon=2$ and 10 respectively. The observation vector $\mathbf{y}$ is perturbed by additive Gaussian noise with 20 $\mathrm{dB}$ SNR. As discussed in Section 3, since co-prime array provides relatively smaller MLAs with acceptable MSLs, it outperforms both nested and random sparse arrays. As wall permittivity increases, the reconstruction error for all architectures increases, since wall multiples increase the ambiguity in the PSF in the form of MSL.

In the second set of experiments, we used a two-dimensional finitedifference time-domain (FDTD) method to realistically simulate the actual received scattered field instead of using our model. This allows for a more realistic experiment, with solid scatterers placed in the scene and no idealized grid assumptions. For this simulation the radar bandwidth is from $1 \mathrm{GHz}$ to $5 \mathrm{GHz}$, imaging a $2.5 \mathrm{~m} \times 2.5 \mathrm{~m}$ square room surrounded by four three-layered walls. A total number of seven metal cylinders are placed inside the room. As with the previous experiment, we start with full MIMO Tx and Rx ULAs with 21 elements each, having inter-element spacing $3 \mathrm{~cm}$.

In the recovery, we first estimate the wall parameters from the measurements, using the approach in Sec. IV-A. As an example, we summarize estimation results in Table I for a 4-by-5 co-prime array.

Using the estimated wall parameters, we reconstruct the image and plot the results in Fig. 6. Specifically, the top row plots illustrate reconstruction results using classical backprojection reconstructioni.e., matched filtering-from (a) the full 21 by 21 array data, (b) a co-prime array, and (c) a nested array. Both sparse array designs use $\widetilde{M}_{r}=4$ and $\widetilde{M}_{t}=5$ elements. The bottom row shows the 

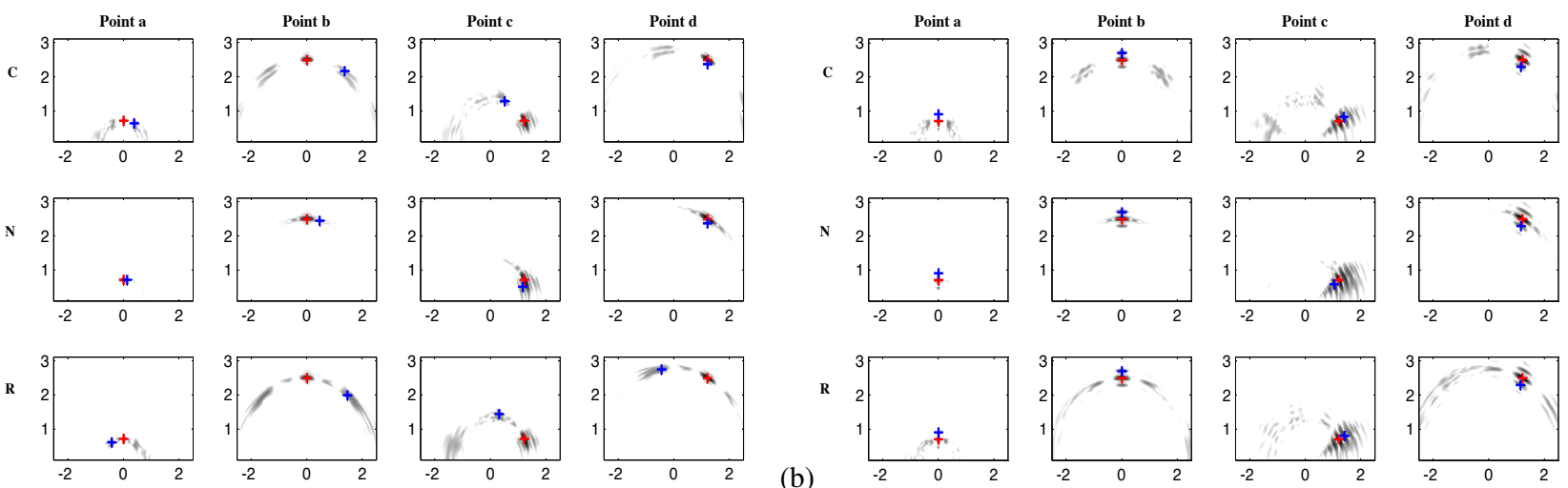

Fig. 3. PSF of co-prime (top), nested (middle) and random (bottom) array designs for a wall with relative permittivity (a) $\epsilon=2$ and (b) $\epsilon=5$ for the four points in Fig. 2. The red cross denotes the location of the peak of the mainlobe and the blue cross the location of the largest sidelobe. The gray intensity level denotes the magnitude of the PSF.

(a)

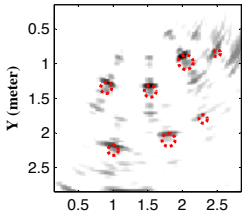

(d)

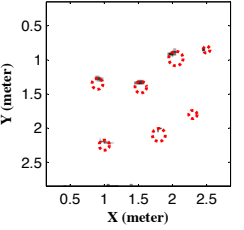

(b)

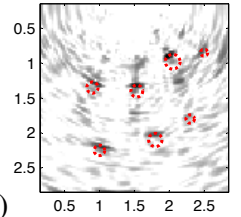

$(\mathrm{e})$

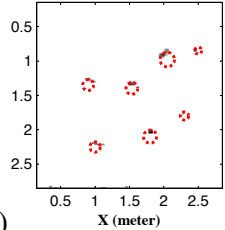

(c)

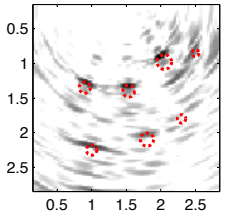

(f)

Fig. 6. Reconstructed image from FDTD simulations. (a)-(c) backprojection, (d)-(e) sparse reconstruction.

reconstruction results for the same array architectures in (d), (e) and (f), respectively, but using sparse reconstruction with the IHT algorithm with $K=45$. In all six plots, the true locations and shapes of the metal objects are marked as red circles. The estimated components from sparse reconstruction are marked as blue crosses.

As evident, the backprojection reconstruction exhibits significant artifacts, even using the full arrays, due to the sidelobes of the PSF. These artifacts, as expected, exagerated using the sparse arrays since the sidelobes increase. The effect is especially pronounced in the co-prime array which exhibits significantly higher sidelobes than the nested array. In contrast, the sparsity-based image reconstruction is robust to the sidelobes due to sparse arrays and is able to recover the image in all three cases.

\section{REFERENCES}

[1] L. C. Potter, E. Ertin, J. T. Parker, and M. Cetin, "Sparsity and compressed sensing in radar imaging," Proceedings of the IEEE, vol. 98, no. 6, pp. $1006-1020$, June 2010.

[2] D. Malioutov, M. Cetin, and A.S. Willsky, "A sparse signal reconstruction perspective for source localization with sensor arrays," IEEE Transactions on Signal Processing, vol. 53, no. 8, pp. 3010-3022, 2005.

[3] P. T. Boufounos, B. Raj, and P. Smaragdis, "Joint sparsity models for broadband array processing," in Proc. SPIE Wavelets and Sparsity XIV, San Diego, CA, August 21-14, 2011.

[4] D.W. Bliss and K.W. Forsythe, "Multiple-input multiple-output (MIMO) radar and imaging: degrees of freedom and resolution," in Conference Record of the Thirty-Seventh Asilomar Conference on Signals, Systems and Computers., 2003, vol. 1, pp. 54-59.

[5] L. Li and J.L. Krolik, "Vehicular MIMO SAR imaging in multipath environments," in IEEE Radar Conference (RADAR), 2011, pp. 989994.

[6] Q. Huang, L. Qu, B. Wu, and G. Fang, "Uwb through-wall imaging based on compressive sensing," IEEE Trans. Geoscience and Remote Sensing, vol. 48, no. 3, pp. 1408-1415, March 2010.

[7] M. Leigsnering, C. Debes, and A.M. Zoubir, "Compressive sensing in through-the-wall radar imaging," in IEEE International Conference on Acoustics, Speech and Signal Processing (ICASSP), May 2011, pp. 4008-4011.

[8] Y.-S. Yoon and M. G. Amin, "Compressed sensing technique for highresolution radar imaging," in SPIE Defense and Security Symposium, 2008, pp. 69681A-69681A

[9] P. P. Vaidyanathan and P. Pal, "Sparse sensing with co-prime samplers and arrays," IEEE Transactions on Signal Processing, vol. 59, no. 2, pp. 573-586, 2011.

[10] P. Pal and P. P. Vaidyanathan, "Nested arrays: a novel approach to array processing with enhanced degrees of freedom," IEEE Trans. Sig. Proc., vol. 58, no. 8, pp. 4167-4181, Aug. 2010

[11] D.L. Donoho, M. Elad, and V.N. Temlyakov, "Stable recovery of sparse overcomplete representations in the presence of noise," IEEE Transactions on Information Theory, vol. 52, no. 1, pp. 6-18, 2006.

[12] T. Blumensath and M. E Davies, "Iterative thresholding for sparse approximations," Journal of Fourier Analysis and Applications, vol. 14, no. 5-6, pp. 629-654, 2008.

[13] F. Soldovieri and R. Solimene, "Through-wall imaging via a linear inverse scattering algorithm," IEEE Geoscience and Remote Sensing Letters, vol. 4, no. 4, pp. 513-517, 2007.

[14] W. Zhang and A. Hoorfar, "Two-dimensional diffraction tomographic algorithm for through-the-wall radar imaging," Progress In Electromagnetics Research B, vol. 31, pp. 205-218, 2011.

[15] W. C. Chew, Waves and fields in inhomogenous media, IEEE press New York, 1995.

[16] P. Protiva, J. Mrkvica, and J. Machac, "Estimation of wall parameters from time-delay-only through-wall radar measurements," IEEE Transactions on Antennas and Propagation, vol. 59, no. 11, pp. 4268-4278, 2011.

[17] Wei Dai and Olgica Milenkovic, "Subspace pursuit for compressive sensing signal reconstruction," Information Theory, IEEE Transactions on, vol. 55, no. 5, pp. 2230-2249, 2009.

[18] D. Needell and J. A. Tropp, "CoSaMP: iterative signal recovery from incomplete and inaccurate samples," Commun. ACM, vol. 53, no. 12, pp. 93-100, Dec. 2010.

[19] R. G. Baraniuk, V. Cevher, M. F. Duarte, and C. Hegde, "Model-based compressive sensing," IEEE Trans. Information Theory, vol. 56, no. 4, pp. 1982-2001, 2010.

[20] V. Cevher, "On accelerated hard thresholding methods for sparse approximation," 2011, vol. 8138, pp. 813811-813811-11.

[21] Anastasios Kyrillidis and Volkan Cevher, "Recipes on hard thresholding methods," in Computational Advances in Multi-Sensor Adaptive Processing (CAMSAP), 2011 4th IEEE International Workshop on. IEEE, 2011, pp. 353-356. 